
\title{
25 Research Soure \\ Bidirectional association of neurodevelopment with growth: A Prospective Cohort Study
}

\section{Xiaotong Wei}

China Medical University

Jiajin Hu

China Medical University

Liu Yang

shenyang women and children health care centre

\section{Ming Gao}

China Medical University

Lin Li

Shengjing Hospital of China Medical University

Ning Ding

China Medical University

\section{Yanan Ma}

China Medical University

Deliang Wen ( $\nabla$ dlwen@cmu.edu.cn )

China Medical University https://orcid.org/0000-0002-3519-2685

\section{Research article}

Keywords: Neurodevelopment, Psychiatric development, Physical growth, Infants

Posted Date: October 19th, 2020

DOI: https://doi.org/10.21203/rs.3.rs-79490/v1

License: (c) (i) This work is licensed under a Creative Commons Attribution 4.0 International License.

Read Full License 


\section{Abstract \\ Background}

Evidence of the association between early life development in neurological and psychiatric with physical growth is controversial, and bidirectional effects have been poorly evaluated. The study aims to use the cross-lagged models and utilize data from the Born in Shenyang Cohort Study to characterize the bidirectional associations of the term-born infants' development in neurological and psychiatric in five domains and physical growth in the early life.

\section{Methods}

This study consists of 688 mother-child dyads from the Born in Shenyang Cohort Study. Infants' anthropometric and development in neurological and psychiatric outcomes were measured twice by the Gesell Development Scale and administered by staff at six and twelve months of age. Cross-lagged analyses and multiple linear regression analyses were used to explore the longitudinal relationships in both directions.

\section{Results}

In terms of longitudinal studies, the negative associations between gross motor and social behavior at six months with BMI $Z$-score at twelve months were found (gross motor: $a \beta=-0.20,95 \% \mathrm{Cl}$ : -0.31 to- 0.09 ; social behavior: $a \beta=-0.23,95 \% \mathrm{Cl}$ : 0.33 to- 0.13$)$. Instead, higher infant $Z$-scored $\mathrm{BMI}$ at six months predicted lower gross motor at twelve months $(a \beta=-0.08,95 \% \mathrm{Cl}:-0.12$ to- 0.04$)$. The only bidirectional development in neurological and psychiatric, gross motor, had a more significant impact on infants' BMI $Z$-score than the reverse $(\beta=-0.21$ vs. $\beta=-0.09 ; P<0.001)$. The similar associations had been found in weight gain velocity.

\section{Conclusion}

We found bidirectional relationships between infants' gross motor with physical growth and suggested the term-born infants, who are on the edge of the developmental danger, should not be overlooked.

\section{Background}

The relationships between physical growth and development in psychiatric and neurological are found; however, the literature for the infants, especially within term-born, is relatively few [1,2]. As far as we know, the outstanding cohort study, which named Avon Longitudinal Study of Parents and Children (ALSPAC), has paid attention to this relationship. They found that physical growth in adults and children were not associated with development in psychiatric and neurological, suggesting that the physical 
development in early life may be a critical period of later mental retardation and neural development [3]. Between birth and one year, bodyweight has triples, body length increases by more than $50 \%$, and brain volume increase to $75 \%$ of an adult's size [4].

Besides, although recent studies have found that bidirectional effects between physical growth and development in psychiatric and neurological, that is, physical growth may be both influencing and responding to the infants', few studies have the analysis of these relationships. On the one hand, physical growth may be a sign of disruption of the critical steps during brain development. The brain and nervous system grows at its fastest rate during this time, such as weight status and weight gain during this process, can have long-term impacts on the brain's developing structure and function [5-8]. Moreover, research addressing children's physical growth on development in psychiatric and neurological has shown controversial findings. Some studies found that weight status has been negatively associated with development in psychiatric and neurological, especially in motor development and cognitive development. However, longitudinal studies confirmed no temporal associations in the population [9-12]. On the other hand, the state of development in psychiatric and neurological young children might also affect physical development $[13,14]$. However, few epidemiologic studies that focus on the effects and have shown controversial findings [15]. Although a comprehensive systematic review study showed infants with better development in psychiatric and neurological demonstrated a greater gain in height and weight gain between 4 and twelve months, the study to explore early development (SEED) showed infants with mental retardation and neurodevelopmental impairments, in particular, would be at greater risk for rapid weight gain or obesity during infancy [16].

The bidirectional relationships between early life development in psychiatric and neurological with physical growth can be affected by numerous confounding factors. As these factors are often complex and interdependent, using independent regression models is cumbersome. In this paper, the study needs to explore the relationships between the growth and development in psychiatric and neurological during the infancy, and investigates causal pathways beginning in early life. The cross-lagged analysis should be used to overcome these problems, which is a powerful statistical approach to address methodological problems.

In this study, we considered to use the cross-lagged models and utilize data from the Born in Shenyang Cohort Study (BISCS) to characterize the bidirectional associations of the term -born infants' development in psychiatric and neurological in five domains (adaptive behavior, gross motor, fine motor, language, and social behavior) and physical growth (Body Mass Index (BMI) Z-score and weight gain velocity) in the early life.

\section{Methods}

\section{Population and study design}

The BISCS was a representative, prospective cohort study conducted by China Medical University between 2017 and 2020. The design of the research has been previously published [17]. The BISCS was 
designed to investigate women and children's health, particularly in development with psychiatric and neurological. In-person visits were conducted with mothers between 13-27 weeks and 28-36 weeks of gestation to record maternal demographic information and collect biological samples. The visits were conducted again at one month, three months, six months and twelve months postpartum to record maternal postnatal data and infant information, and use the Gesell Developmental Scale to assess the infant cognitive and neurodevelopmental levels six months and twelve months old. Children born to mothers before 36 weeks gestation, with severe complications during pregnancy, diseases in the nervous system around birth ,as part of a multiple pregnancies (e.g., twins, triplets), or without complete baseline and follow-up data were excluded. As Figure 1, a final cohort of 688 women was completed at delivery, six months postpartum $(n=459)$, and twelve months postpartum $(n=449)$. There was no difference found in maternal and infant demographic information between attrite participants and those with complete information (see Table. S1).

\section{Measures}

\section{Anthropometric outcomes}

The weight and length at birth of the infants were recorded from the medical records, then at six months old (mean: $6.87 \pm 1.27$ ) and twelve months postpartum (mean: $11.67 \pm 1.41$ ) by BISCS staff, according to a standard protocol. The weight and length were measured with a digital scale and a stadiometer while children were wearing no shoes and light clothes (Seca 416 and $376+$; Seca corporation, Hamburg, Germany). Birth $Z$-score and $Z$-scored BMI for age and sex-specific weight for age (WFA) were calculated based on the World Health Organization (WHO) child growth reference [18]. Weight gain velocity was calculated as the change in WFA $Z$-score from birth to six months and six months to twelve months [19].

\section{Infants' development in neurological and psychiatric}

Participants in this birth cohort were administered the Gesell Development Scale (GDS), which has been used extensively after being translated and standardized (Beijing Children's Health Care Institute, 1985) [20]. The Gesell Development Scale is used to evaluate the function of the central nervous system and identify defects in the neuromuscular or sensory system, which consists of five domains, including the adaptive domain (coordination, imitation, object recovery, discrimination and perception), gross motor domain (changes in posture, head balance and behavioral observations of standing, sitting and walking) and fine motor domain (ability to hold objects with fingers), language domain (vocabulary comprehension and dialogue skills), and social domain (social habits, reactions to persons, autonomy and independence). Developmental quotient (DQ) is defined as the quotient between developmental age and actual age, and the DQ of each child was calculated for each specific domain. Higher developmental quotient (DQ) scores mean higher cognitive levels [21]. Two well-trained pediatricians from the Shengjing Hospital of China Medical University assessed all participants to maximize reliability. They assessed all children in their assigned domain in order to avoid inter-examiner variability.

\section{Covariates}


Based on previous research [22-25], other variables were used to describe maternal and infants' demographic characteristics and control for confounding variables. These variables were collected from hospital records (maternal age, maternal pre-pregnancy BMI [26], gestational weight gain (GWG), delivery mode, parity, child sex, gestational weeks, and birth weight) and questionnaires (maternal education, annual family income, the primary caregiver, mode of infant feeding, the introduction of solid foods at six months and micronutrients supplementation (Iron, Vitamin B12 and Zinc) at six months). Only $0.85 \%$ of women smoked and $1.15 \%$ of women were drinking during pregnancy, so this variable was not included. We calculated the GWG rate (kg/week) as total GWG divided by the number of gestational weeks at delivery. Based on the IOM, above optimal weight gains are above $16 \mathrm{~kg}$ for mothers with normal weight and more than $11.5 \mathrm{~kg}$ for overweight mothers, respectively [27,28]. We categorized the mode of infant feeding as: formula feeding only, mixed breast and formula, and breast milk feeding only. Micronutrient supplementation (Iron, Vitamin B12 and Zinc) was treated as a dichotomized variable (yes/no). To ensure data integrity, wholly conditional specification multiple imputation $(m=20)$ was used to impute missing data.

\section{Statistical analyses}

\section{Longitudinal analyses}

Multiple linear regression analyses were fitted to assess the association of infants' BMI $Z$-score and weight gain velocity with the development in neurological and psychiatric status at the age of six and twelve months. The analyses used the repeated measured weight status and development in neurological and psychiatric variables at two-time points. The analyses were adjusted for all the above confounding factors. Furthermore, the associations between development in neurological and psychiatric status at six months and weight status at twelve months were additionally adjusted for weight status at baseline (six months) to study whether development in neurological and psychiatric status predicted the change in infants' $Z$-scored BMI or weight gain velocity, and vice versa. Linear regression analyses were run using SPSS version 20.0 (SPSS 20.0, [2011], IBM).

\section{Cross-lagged analyses}

This cross-lagged analysis included confounders, stability effects, cross-sectional associations, longitudinal associations, and cross-lagged associations. Firstly, we examined the stability model, which only included the cross-sectional analysis, with confounding factors regressed at two baselines. Then, based on the first step, we entered the lagged association between infant BMI $Z$-score and weight gain velocity at six months with development in neurological and psychiatric status at twelve months, and the lagged association between development in neurological and psychiatric status at six months with infant BMI $Z$-score and weight gain velocity at twelve months. Finally, the above two lag associations entered the whole model at the same time. The above relationships were adjusted for all the above confounding factors. Besides, the cross-lagged associations were adjusted for weight status at baseline (six months) or development in neurological and psychiatric status at baseline (six months). The cross-lagged analyses were conducted with Mplus, version 7.11. 


\section{Results}

\section{Participants characteristics}

Characteristics at the follow-up at six and twelve months of age were shown (Table. S1, see Supplemental Materials). Among eligible mother-offspring pairs, the mean (SD) age of the recruited mothers was $30.4 \pm 3.9$ years, and about a quarter of mothers were overweight. 459 infants (234[51.1\%] were boys, 225 [48.9\%]) were girls who assessed at six months. At six months and twelve months, the mean infants' BMI $Z$-score were $0.16 \pm 0.97$ and $0.23 \pm 1.05$, respectively. Nearly one-fourth of infants went through rapid weight gain, and at six months and twelve months, 47 (10.2\%) and 51 (11.4\%) were overweight, respectively. The infant average DQ scores in domains of adaptive behavior, gross motor, fine motor, language, and social behavior were $97.9,100.3,94.1,85.9$, and 93.2 at six months, respectively. Furthermore, the mean scores were 103.3, 101.2, 104.8, 94.9, and 112.7 at twelve months, respectively.

\section{Longitudinal analyses}

As shown in Table. 1 and Table. S2 (see Supplemental Materials), the linear regression analysis evaluated the associations between development in neurological and psychiatric status at the age of six months and $Z$-scored BMI at twelve months. There were inverse associations between infants' two skills (gross motor and social behavior) at six months with BMI $Z$-scores at twelve months (gross motor: $\mathrm{a} \beta=-0.20,95 \% \mathrm{Cl}:-0.31$ to- $0.09, P=0.002$; social behavior: $\mathrm{a} \beta=-0.23,95 \% \mathrm{Cl}:-0.33$ to- $0.13, P=0.005)$. No effect of the other three development in neurological and psychiatric skills was observed. Similar associations had been found between the DQ scores at six months and the risk of rapid weight gain at twelve months.

As shown in Table. 2, the linear regression analysis assessed the associations between $Z$-scored $\mathrm{BMI}$ at the age of six months and development in neurological and psychiatric status at twelve months (Table. 2). A higher infant $Z$-scored BMI at six months was associated with a lower neurological development level in the gross motor domain at twelve months, and all associations were attenuated but still significant after adjusting for covariates $(a \beta=-0.08,95 \% \mathrm{Cl}:-0.12$ to- $0.04, P=0.03)$. However, $Z$-scored $\mathrm{BMl}$ at six months showed a negative correlation with social behavior skill at twelve months, which tends to disappear when considering social behavior skill at six months as a confounding variable(model 2: $\mathrm{a} \beta=-0.08,95 \% \mathrm{Cl}:-0.11$ to- $0.05, P=0.03$; model 3 : $a \beta=-0.05,95 \% \mathrm{Cl}:-0.08$ to $0.00, P=0.06)$. Like $Z$-scored $\mathrm{BMI}$ results, the relationships between weight gain velocity at six months with development in neurological and psychiatric status at twelve months were performed in Table. S3 (see Supplemental Materials).

\section{Cross-lagged analyses}

The cross-lagged models of bidirectional associations between infant neurological development levels and $Z$-scored BMI at six and twelve months were shown in Fig. 2. All cross-lagged analyses demonstrated SRMR and RMSEA close to zero, and TLI and CFI were above 0.9, which means the degrees 
of fitting were good. An adverse association in both directions between gross motor and $Z$-scored BMI was observed; however, the likelihood ratio test confirmed that the more vital direction was from the neurological development to infants' BMI $Z$-score. The negative relationship of social behavior at six months of age with BMI $Z$-score at 1 year of age did not differ statistically from the association in the opposite direction. Similar to $Z$-scored BMI results, the cross-lagged models of bidirectional associations between weight gain velocities with development in neurological and psychiatric status were performed in Fig. 3.

\section{Discussion}

To the best of our knowledge, the current study is one of the few to explore the bidirectional associations between development in neurological and psychiatric, as assessed by the Gesell Development Scale, and physical growth among term-born infants. Our birth cohort study suggests that the negative associations between gross motor and social behavior at six months with weight status at twelve months. Regarding the opposite direction of relationship, higher infant $Z$-scored BMI at six months predicted lower neurological development level in the gross motor at twelve months. Gross motor performed a bidirectional relationship, although neurological development level had a more significant effect on infants' BMI $Z$-score than the opposite. The similar associations had been found in weight gain velocity.

To motor abilities, our findings are consistent with previous studies demonstrating that gross motor skills were negatively associated with infant physical growth in both directions [29]. However, Schmidt et al. found minimal correlations between motor development and weight status, suggesting that weight status and motor milestones are mostly independent of one another [30]. There are several possible reasons for these difference. First, the samples were analyzed in different age groups. Most recent studies concentrate on exploring relationships in preschool children or school-aged children, whereas the participants in our study were infants, especially within term-born infants. Second, differences may have arisen due to the fact that different tools measured the participants. The current study evaluated motor skills using the GDS, which were in line with motor milestones and were dependent on the age interval of the infants. However, this scale is still heterogeneous with other measurement tools. Potential mechanisms for these effects include the possibility that infants with poor gross motor performance are frequently associated with biomechanical problems and morphological constraints on tasks involving changes in overweight/obese status and fat mass [31]. However, Gentier and colleagues suggested that a deficit in childhood motor skills should be examined in a broader sense, rather than a mechanical interpretation. For example, it is plausible that the reduced physical activity and the lack of decisionmaking, planning, and control functions in infants with poor motor ability are causal factors in obese status, suggesting that impaired motor development is also related to children obesity [12]. Therefore, additional exploratory analyses to determine the bidirectional associations between motor domains and weight status should be conducted.

Consistent with our results, previous studies observed the relationships between social skills and infant's physical development [32]. According to the Infant Research framework [33], infant social communication 
abilities develop in dynamic functional interactions between mothers and infants. Ostensibly, it should be recognized that the relationship between mother and infant is bidirectional and may influence infant clues' accuracy. As infants grow, they use communicate abilities to express wants or dislike certain foods [34]. Suppose infants have poor social skills, such as infrequent laughter, reduced focus, shared attention, and/or unclear signals to articulate their needs (including the cues of full or hungry). In that case, it may preclude their potential development of caregivers' feeding responsiveness [35]. Scholars propose that maternal responsiveness to their children's expressions, such as appetite, hunger, and satiety is essential developing a healthy diet and could play an essential role in offspring weight status [32]. Besides, it is generally known that language and social competence can be simultaneously discussed as previous studies have demonstrated that children's social and language development are synchronized mainly[36]; However, no relationship between language ability and body weight was found in our study. Additional research is needed to investigate further the potential contribution of children's social communication skills and language ability with maternal factors.

Our prospective study provided a unique opportunity to explore the bidirectional relationships between infants' development in neurological and psychiatric and physical growth and had attempted to analyze the associations of mutual prediction, which have been of little attention to date. However, there are also several limitations. First, our study is limited in sample size and follow-up time, then might weakly predict later attainment. Nevertheless, physical and neurological development in early life is irreversible ${ }^{3}$. Based on this study, long-term follow-up data will be used to verify our research results further. Second, Confounding factors, such as feeding style (responding style, authoritative feeding, etc.), breastfeeding (bottle or exclusive breastfeeding) were not fully adjusted.

\section{Conclusion}

Our works suggest that the development within term-born infants should not be overlooked, but only concerned children with developmental disabilities are incomplete. Strengthen knowledge education for caregivers and improve the physical examination system for infants and young children could avoid becoming a hidden danger in developmental delays.

\section{Abbreviations}

ALSPAC: Avon Longitudinal Study of Parents and Children; SEED:The study to explore early development; BISCS:Born in Shenyang Cohort Study; BMI:Body Mass Index; WFA:Weight for age ; WHO:World Health Organization ; GDS:Gesell Development Scale; DQ:Developmental quotient ; GWG:Gestational weight gain ; Wt:weight.

\section{Declarations}

\section{Ethics approval and consent to participate}


All procedures were approved by the medical ethics committee of China Medical University (application number: 71774173). The mothers were informed of the project aims and requirements. We obtained written informed consent from the mothers prior to enrollment.

\section{Consent for publication}

Not applicable.

\section{Availability of data and materials}

The datasets used and/or analyzed during the current study are available from the corresponding author on reasonable request

\section{Competing interests}

The authors declare no potential conflicts of interest.

\section{Funding}

The study was supported by China Postdoctoral Science Foundation (2019M661179).

\section{Author contributions}

X.W., J.H. and D.W conceptualized the study concept and design. X.W, M.G, and Y.M conducted research; X.W, and J.H. analyzed the data; and X.W., L.Y., L.L., and J.H. drafted the manuscript. All authors were involved in writing the paper and had final approval of the submitted and published versions.

\section{Acknowledgements}

We thank all the participants who took part in our study, and interviewers, nurses, computer and laboratory technicians, clerical workers, research scientists, volunteers, managers, and receptionists of the BISCS study for their supports and dedications. The study was supported by China Postdoctoral Science Foundation (2019M661179).

\section{Authors' Information}

${ }^{1}$ Institute of Health Sciences, China Medical University, Shenyang 110122, Liaoning, China; ${ }^{2}$ Department of obstetrics and gynecology, Shenyang Women and Children Health Care Centre, Shenyang, Liaoning, China; ${ }^{3}$ Department of developmental pediatrics, Shengjing Hospital of China Medical University $\mathbb{}$ Shenyang, Liaoning, China $\otimes^{4}$ Curriculum and teaching research office, Research Center of Medical Education, China Medical University, Shenyang, Liaoning, China; ${ }^{5}$ Department of Epidemiology and Health Statistics, School of Public Health, China Medical University, Shenyang 110122, Liaoning, China;

\section{References}


1. Richards M, Hardy R, Kuh D, Wadsworth ME. Birth weight and cognitive function in the British 1946 birth cohort: longitudinal population based study. BMJ. 2001;322:199-203.

2. Shenkin SD, Starr JM, Deary IJ. Birth weight and cognitive ability in childhood: a systematic review. Psychol Bull. 2004;130:989-1013.

3. Gale CR, O'Callaghan FJ, Bredow M, Martyn CN. Parents ALSo, Team CS. The influence of head growth in fetal life, infancy, and childhood on intelligence at the ages of 4 and 8 years. Pediatrics. 2006;118:1486-92.

4. World Health Organization. World Health Organization child growth standards: growth velocity based on weight, length and head circumference: methods and development. World Health Organization 2009.

5. Adelantado-Renau M, et al. Inflammatory biomarkers and brain health indicators in children with overweight and obesity: the activebrains project. Brain Behavior Immunity. 2019;81:588-97.

6. Nanda S, Menon R, Kumari S, et al. Visual-verbal paired associate memory in mild cognitive impairment: A neuropsychological and brain volumetric study. J Neurol Sci. 2019;405:16-7.

7. Grantham-McGregor S, et al. Developmental potential in the first 5 years for children in developing countries. The Lancet. 2007;369:60-70.

8. Thompson RA, Nelson CA. Developmental science and the media: Early brain development. Am Psychol. 2001;56:5-15.

9. Camargos ACR, Mendonça VA, Andrade CA, d., Oliveira KSC, Lacerda ACR. Overweight and obese infants present lower cognitive and motor development scores than normal-weight peers. Res Dev Disabil. 2016;59:410-6.

10. Frey GC, Chow B. Relationship between BMI, physical fitness, and motor skills in youth with mild intellectual disabilities. International Journal of Obesity. 2006;30:861-7.

11. Mond JM, Stich H, Hay PJ, Kraemer A, Baune BT. Associations between obesity and developmental functioning in pre-school children: a population-based study. International Journal of Obesity. 2007;31:1068-73.

12. Gentier I, et al. Fine and gross motor skills differ between healthy-weight and obese children. Res Dev Disabil. 2013;34:4043-51.

13. Slining M, Adair LS, Goldman BD, Borja JB, Bentley M. Infant Overweight Is Associated with Delayed Motor Development. The Journal of Pediatrics. 2010;157:20-5.e21.

14. Oudgenoeg-Paz O, Mulder H, Jongmans MJ, van der Ham IJM, Van der Stigchel. S. The link between motor and cognitive development in children born preterm and/or with low birthweight: A review of current evidence. Neuroscience Biobehavioral Reviews. 2017;80:382-93.

15. Levy SE, et al. Relationship of Weight Outcomes, Co-Occurring Conditions, and Severity of Autism Spectrum Disorder in the Study to Explore Early Development. The Journal of Pediatrics. 2019;205:202-9. 
16. Kral TVE, et al. Early life influences on child weight outcomes in the Study to Explore Early Development. Autism. 2018;23:954-62.

17. Hu J, et al. Association of Total and Trimester-Specific Gestational Weight Gain Rate with Early Infancy Weight Status: A Prospective Birth Cohort Study in China. Nutrients. 2019;11:280.

18. GROUP WMGRS. WHO Child Growth Standards based on length/height, weight and age. Acta Pædiatrica. 2006;Suppl 450:76-85.

19. De Onis M, Lobstein T. Defining obesity risk status in the general childhood population: Which cutoffs should we use? International Journal of Pediatric Obesity. 2010;5:458-60.

20. Jin X, et al. "Care for Development" Intervention in Rural China: A Prospective Follow-up Study. J Dev Behav Pediatr. 2017;28:213-8.

21. Perera F, et al. Benefits of Reducing Prenatal Exposure to Coal-Burning Pollutants to Children's Neurodevelopment in China. Environmental Health Perspectives 200; 116: 1396-1400.

22. Tian Y, et al. Influencing factors of the neurodevelopment of high-risk infants. General Psychiatry. 2018;31:e100034.

23. Sanchez CE, et al. Maternal pre-pregnancy obesity and child neurodevelopmental outcomes: a metaanalysis. Obes Rev. 2018;19:464-84.

24. Yeung EH, Sundaram R, Ghassabian A, Xie Y. \& Buck Louis, G. Parental Obesity and Early Childhood Development. Pediatrics 2017; 139.

25. Linsell L, Malouf R, Morris J, Kurinczuk JJ, Marlow N. Prognostic Factors for Poor Cognitive Development in Children Born Very Preterm or With Very Low Birth Weight. JAMA Pediatrics. 2015;169:1162.

26. Appropriate body-mass. index for Asian populations and its implications for policy and intervention strategies. The Lancet. 2004;363:157-63.

27. Delphina $\mathrm{G}$, et al. Late-pregnancy dysglycemia in obese pregnancies after negative testing for gestational diabetes and risk of future childhood overweight: An interim analysis from a longitudinal mother-child cohort study. PLOS Medicine. 2018;15(10):e1002681.

28. Rasmussen KM, Catalano PM, Yaktine AL. New guidelines for weight gain during pregnancy: what obstetri- cian/gynecologists should know. Current Opinion in Obstetrics Gynecology. 2009;21:521-6.

29. D'Hondt $E$, et al. A longitudinal analysis of gross motor coordination in overweight and obese children versus normal-weight peers. International Journal of Obesity. 2012;37:61-7.

30. Schmidt Morgen C, et al. Timing of motor milestones achievement and development of overweight in childhood: a study within the Danish National Birth Cohort. Pediatric Obesity. 2014;9:239-48.

31. Chivers P, Larkin D, Rose E, Beilin L, Hands B. Low motor performance scores among overweight children: Poor coordination or morphological constraints? Hum Mov Sci. 2013;32:1127-37.

32. Shloim N, Shafiq I, Blundell-Birtill P, Hetherington MM. Infant hunger and satiety cues during the first two years of life: Developmental changes of within meal signalling. Appetite. 2018;128:303-10. 
33. Fadda R, Lucarelli L. Mother-Infant and Extra-Dyadic Interactions with a New Social Partner: Developmental Trajectories of Early Social Abilities during Play. Frontiers in Psychology 2017; 8.

34. DiSantis KI, Hodges EA, Johnson SL, Fisher JO. The role of responsive feeding in overweight during infancy and toddlerhood: a systematic review. International Journal of Obesity. 2011;35:480-92.

35. Gartstein MA, Crawford J, Robertson CD. Early Markers of Language and Attention: Mutual Contributions and the Impact of Parent-Infant Interactions. Child Psychiatry Hum Dev. 2007;39:926.

36. Igualada A, Bosch L, Prieto P. Language development at 18 months is related to multimodal communicative strategies at 12 months. Infant Behavior Development. 2015;39:42-52.

\section{Tables}

Table 1 Longitudinal associations between neurodevelopment and BMI $Z$-scores at 6 and 12 months of age $^{1}$

\begin{tabular}{|c|c|c|c|}
\hline Outcomes at 12 months & Predictors at 6 months & & $\beta(95 \% \mathrm{Cl})$ \\
\hline \multirow[t]{15}{*}{ Infant Z-scored BMI } & Adaptive behavior & Model 1 & $-0.06(-0.20,0.08)$ \\
\hline & & Model 2 & $-0.04(-0.18,0.10)$ \\
\hline & & Model 3 & $-0.03(-0.19,0.13)$ \\
\hline & Gross motor & Model 1 & $-0.54(-0.67,-0.41)$ * \\
\hline & & Model 2 & $-0.44(-0.58,-0.30)$ * \\
\hline & & Model 3 & $-0.20(-0.31,-0.09)$ * \\
\hline & Fine motor & Model 1 & $-0.03(-0.15,0.09)$ \\
\hline & & Model 2 & $-0.03(-0.16,0.11)$ \\
\hline & & Model 3 & $-0.01(-0.05,0.04)$ \\
\hline & Language & Model 1 & $-0.03(-0.13,0.07)$ \\
\hline & & Model 2 & $-0.03(-0.12,0.07)$ \\
\hline & & Model 3 & $-0.02(-0.10,0.06)$ \\
\hline & Social behavior & Model 1 & $-0.35(-0.48,-0.23)$ * \\
\hline & & Model 2 & $-0.31(-0.41,-0.21)$ * \\
\hline & & Model 3 & $-0.23(-0.33,-0.13)$ * \\
\hline
\end{tabular}

${ }^{1} \mathrm{n}=449$. $\mathrm{n}$ varied from $1.7 \%$ to $2.8 \%$ in each regression because the complete data for each subscale of the Gesell Development Scale were varied. 
Model 1: adjusted for basic information; Model 2: model 1+ maternal pre-pregnancy BMI, gestational weight gain, delivery mode, gestational weeks, birth weight $Z$-score, mode of infant feeding, introduction of solid foods and micronutrients supplementation; Model 3: model 2+ Infant Z-scored BMI at 6 months in the neurodevelopment- BMI relationships, or neurodevelopment scores at 6 months in the BMI neurodevelopment relationships.

*Statistically significant.

Table 2 Longitudinal associations between BMI $Z$-scores and neurodevelopment at 6 and 12 months of age $^{1}$

\begin{tabular}{|c|c|c|c|}
\hline Outcomes at 12 months & Predictors at 6 months & & $\beta(95 \% \mathrm{Cl})$ \\
\hline \multirow[t]{3}{*}{ Adaptive behavior } & Infant Z-scored BMI & Model 1 & $-0.01(-0.03,0.01)$ \\
\hline & & Model 2 & $-0.01(-0.02,0.01)$ \\
\hline & & Model 3 & $-0.01(-0.02,0.01)$ \\
\hline \multirow[t]{3}{*}{ Gross motor } & & Model 1 & $-0.29(-0.36,-0.22)$ * \\
\hline & & Model 2 & $-0.19(-0.29,-0.09)$ * \\
\hline & & Model 3 & $-0.08(-0.12,-0.04)^{\star}$ \\
\hline \multirow[t]{3}{*}{ Fine motor } & & Model 1 & $-0.04(-0.11,0.03)$ \\
\hline & & Model 2 & $-0.02(-0.06,0.03)$ \\
\hline & & Model 3 & $-0.02(-0.04,0.01)$ \\
\hline \multirow[t]{3}{*}{ Language } & & Model 1 & $-0.02(-0.04,0.01)$ \\
\hline & & Model 2 & $-0.02(-0.04,0.01)$ \\
\hline & & Model 3 & $-0.02(-0.03,0.01)$ \\
\hline \multirow[t]{3}{*}{ Social behavior } & & Model 1 & $-0.09(-0.12,-0.08)^{*}$ \\
\hline & & Model 2 & $-0.08(-0.11,-0.05)^{*}$ \\
\hline & & Model 3 & $-0.05(-0.08,0.00)$ \\
\hline
\end{tabular}

${ }^{1} \mathrm{n}=449$. $\mathrm{n}$ varied from $1.7 \%$ to $2.8 \%$ in each regression because the complete data for each subscale of the Gesell Development Scale were varied.

Model 1: adjusted for basic information; Model 2: model 1+ maternal pre-pregnancy BMI, gestational weight gain, delivery mode, gestational weeks, birth weight $Z$ score, mode of infant feeding, introduction of solid foods and micronutrients supplementation; Model 3: model 2+ Infant Z-scored BMI at 6 months 
in the neurodevelopment- BMI relationships, or neurodevelopment scores at 6 months in the BMI neurodevelopment relationships.

*Statistically significant.

\section{Figures}

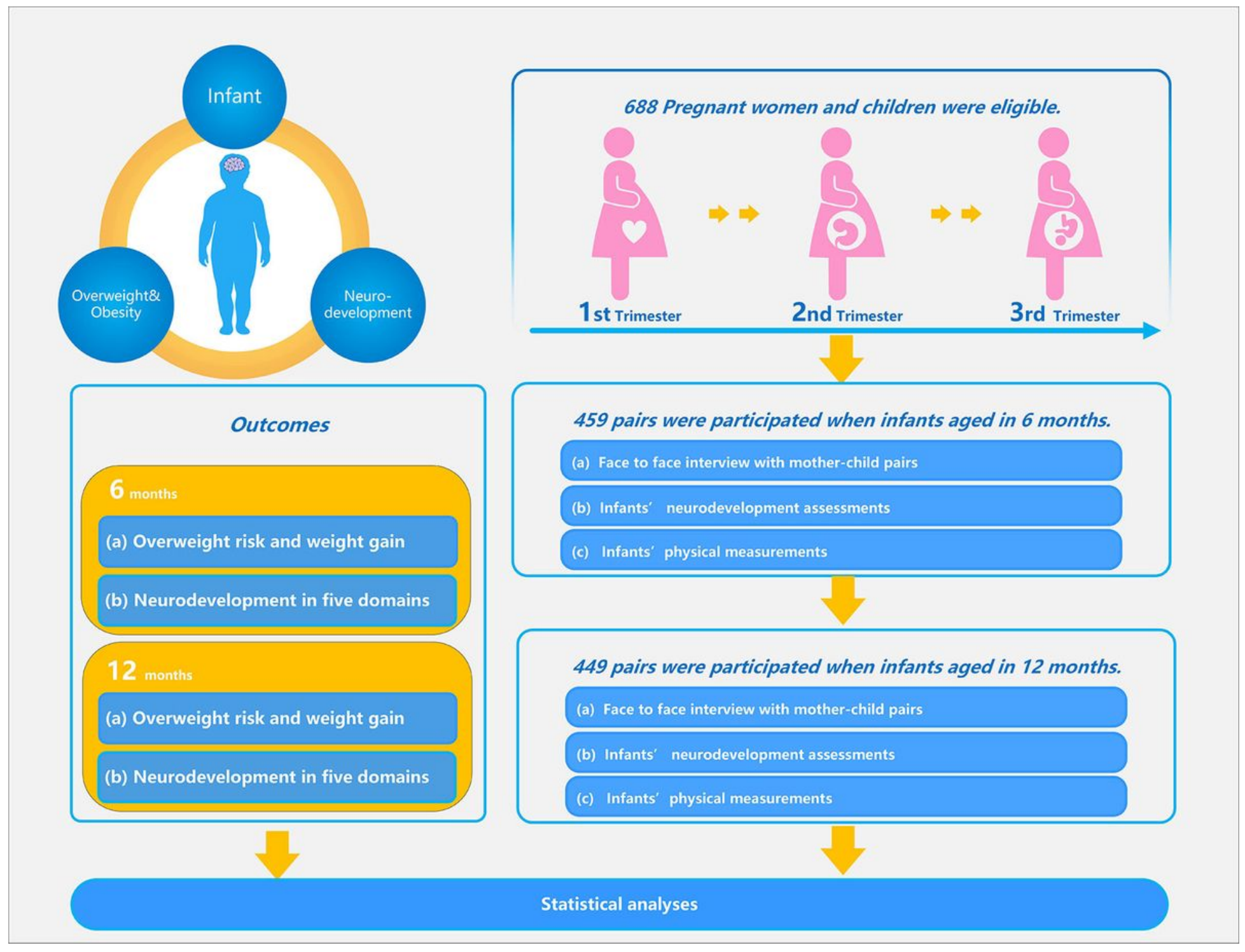

\section{Figure 1}

Flowchart of recruitment and research 
A

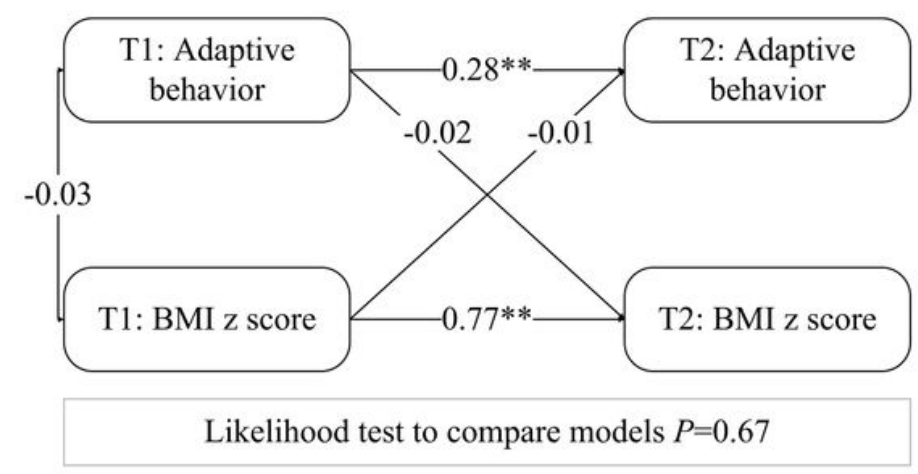

C

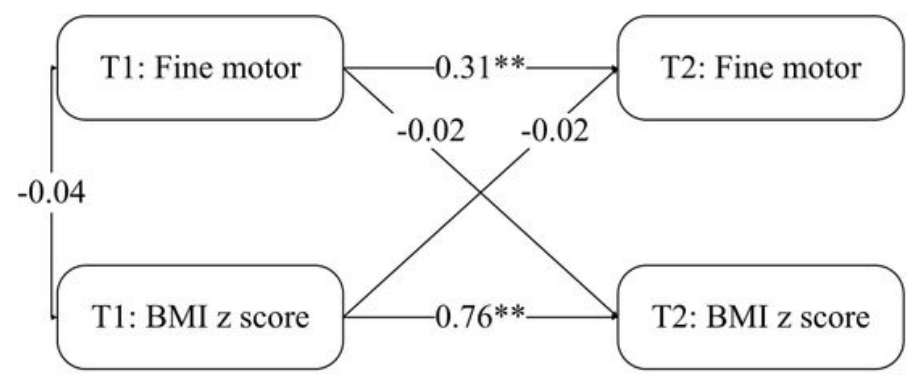

Likelihood test to compare models $P=0.58$

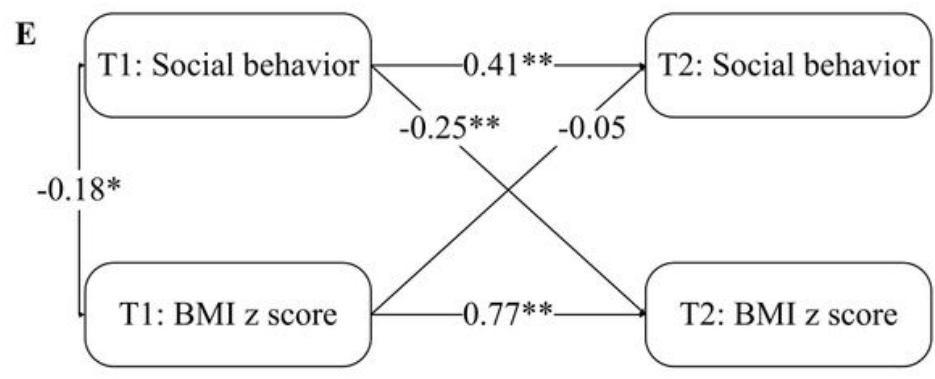

Likelihood test to compare models $P=0.03$
B

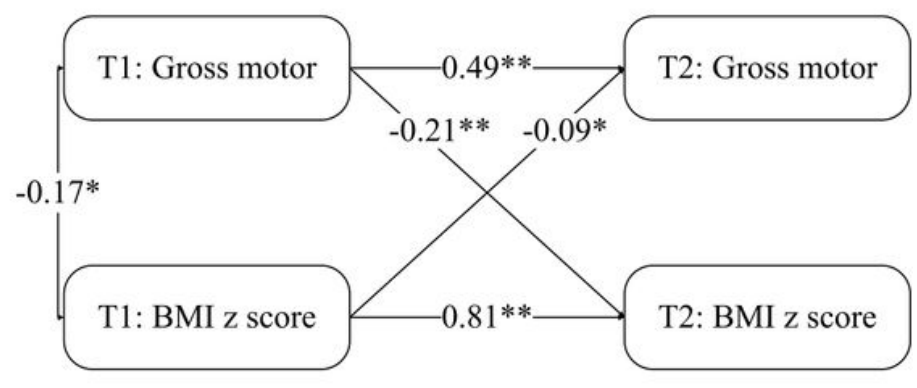

Likelihood test to compare models $P<0.001$

D

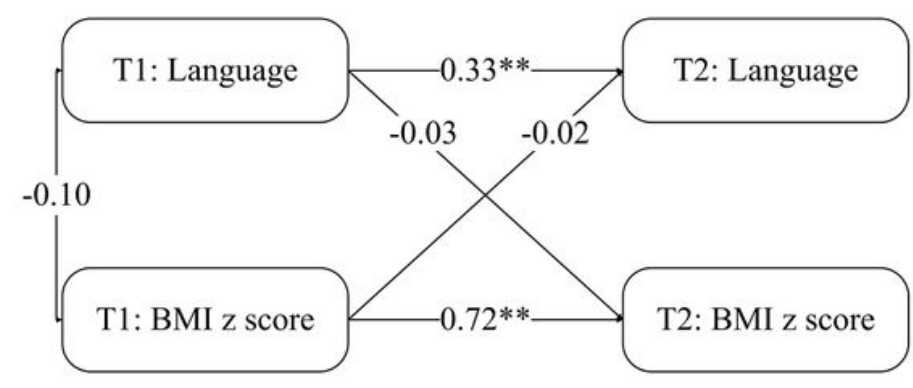

Likelihood test to compare models $P=0.56$

\section{Figure 2}

Cross-lagged model of the associations between infant development in neurological and psychiatric levels and $\mathrm{Z}$-scored BMI at 6 and 12 months $(\mathrm{N}=449)$. The values represent $\beta$-regression coefficients and adjusted for confounding variables. The model fit well among the five models, and ranged from: $X 2=$ 6.86-20.98, RMSEA $=0.02-0.05, \mathrm{CFI}=0.96-1.00, \mathrm{TLI}=0.98-1.00, \mathrm{SRMR}=0-0.02$. BMI: body mass index. ${ }^{\mathrm{P}}$ $<0.05, * * P<0.001$. 
A

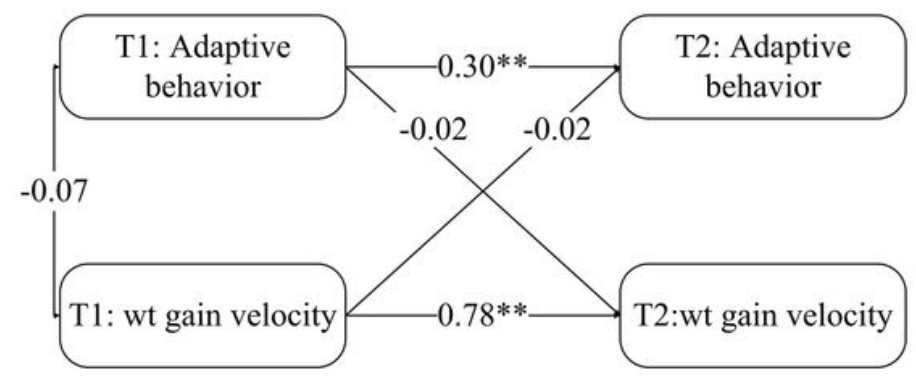

Likelihood test to compare models $P=0.61$

C

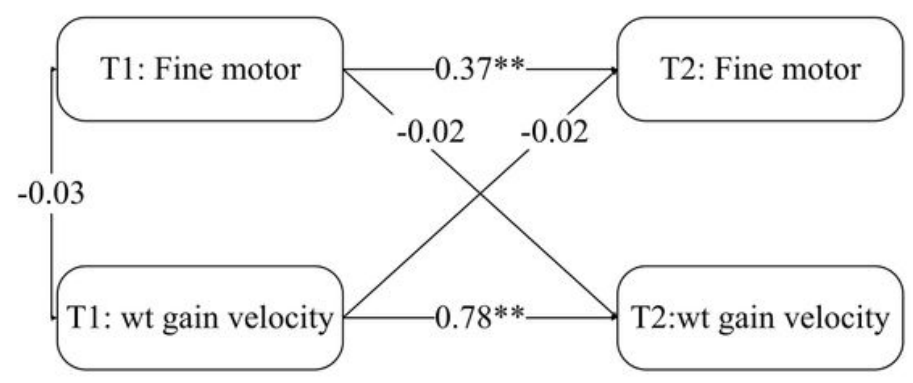

Likelihood test to compare models $P=0.63$

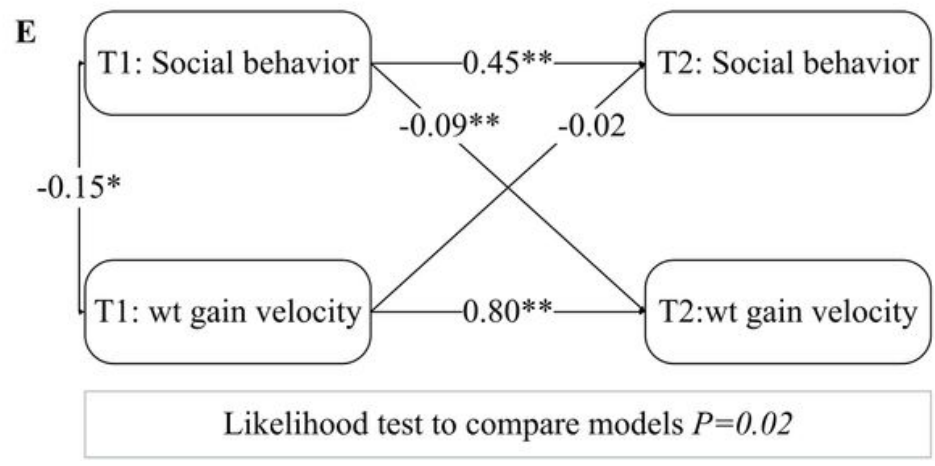

B

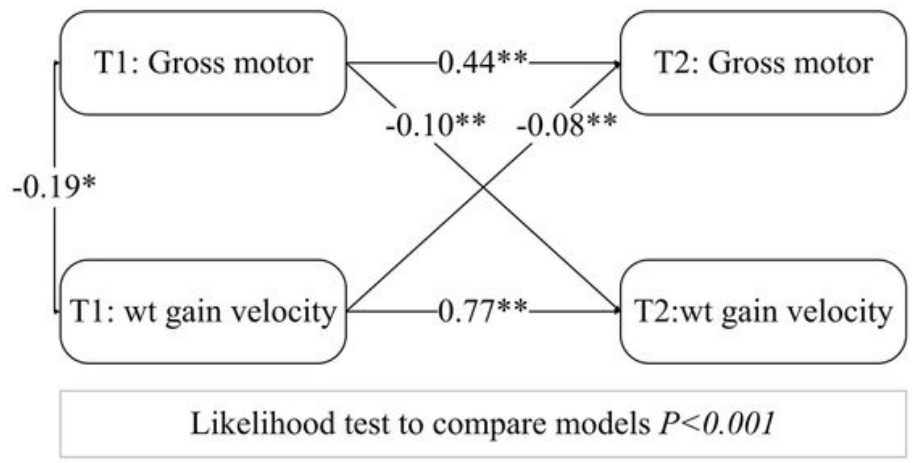

D

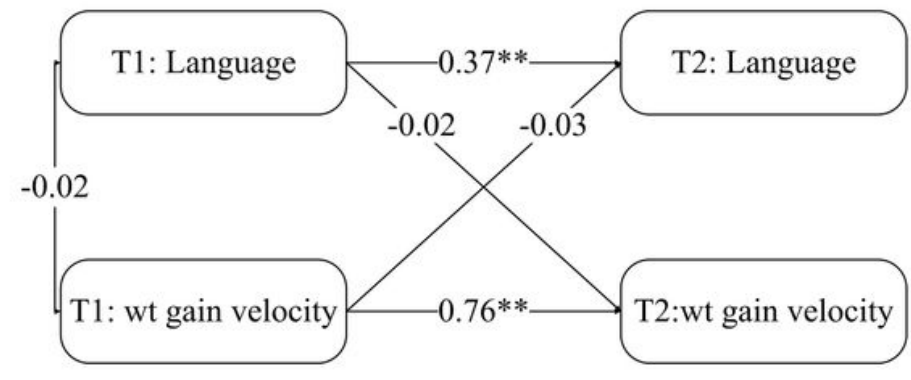

Likelihood test to compare models $P=0.49$

\section{Figure 3}

Cross-lagged model of the associations between infant development in neurological and psychiatric levels and weight gain velocity at 6 and 12 months $(N=449)$. The values represent $\beta$-regression coefficients and adjusted for confounding variables. The model fit well among the five models, and ranged from: $\mathrm{X} 2=8.86-27.98, \mathrm{RMSEA}=0.01-0.04, \mathrm{CFI}=0.97-1.00, \mathrm{TLI}=0.95-1.00, \mathrm{SRMR}=0.00-0.02$. wt: weight. ${ }^{*} \mathrm{P}<0.05,{ }^{\star *} \mathrm{P}<0.001$.

\section{Supplementary Files}

This is a list of supplementary files associated with this preprint. Click to download. 
- WEINeurodevelopmentandAdipositySupplementaltable1.doc

-WEINeurodevelopmentandAdipositySupplementaltable2.docx

-WEINeurodevelopmentandAdipositySupplementaltable3.docx 